\title{
A naturally occurring InDel variation in BraA.FLC.b (BrFLC2) associated with flowering time variation in Brassica rapa
}

\author{
Jian $\mathrm{Wu}^{1 \dagger}$, Keyun Wei ${ }^{1 \dagger}$, Feng Cheng ${ }^{1}$, Shikai Li², Qian Wang ${ }^{2}$, Jianjun Zhao ${ }^{3}$, Guusje Bonnema ${ }^{3}$
} and Xiaowu Wang ${ }^{1 *}$

\begin{abstract}
Background: Flowering time is an important trait in Brassica rapa crops. FLOWERING LOCUS C (FLC) is a MADS-box transcription factor that acts as a potent repressor of flowering. Expression of FLC is silenced when plants are exposed to low temperature, which activates flowering. There are four copies of $F L C$ in $B$. rapa. Analyses of different segregating populations have suggested that BraA.FLC.a (BrFLC1) and BraA.FLC.b (BrFLC2) play major roles in controlling flowering time in B. rapa.

Results: We analyzed the BrFLC2 sequence in nine B. rapa accessions, and identified a 57-bp insertion/deletion (InDel) across exon 4 and intron 4 resulting in a non-functional allele. In total, three types of transcripts were identified for this mutated BrFLC2 allele. The InDel was used to develop a PCR-based marker, which was used to screen a collection of 159 B. rapa accessions. The deletion genotype was present only in oil-type B. rapa, including ssp. oleifera and ssp. tricolaris, and not in other subspecies. The deletion genotype was significantly correlated with variation in flowering time. In contrast, the reported splicing site variation in BrFLC1, which also leads to a non-functional locus, was detected but not correlated with variation in flowering time in oil-type B. rapa, although it was correlated with variation in flowering time in vegetable-type B. rapa.

Conclusions: Our results suggest that the naturally occurring deletion mutation across exon 4 and intron 4 in BrFLC2 gene contributes greatly to variation in flowering time in oil-type B. rapa. The observed different relationship between BrFLC1 or BrFLC2 and flowering time variation indicates that the control of flowering time has evolved separately between oil-type and vegetable-type B. rapa groups.
\end{abstract}

\section{Background}

Brassica rapa is a genus comprising a variety of vegetables such as Chinese cabbage (ssp. pekinensis), pak choi (ssp. chinensis), and turnip (ssp. rapa) as well as oil crops including turnip rape (ssp. oleifera) and sarson (ssp. tricolaris). Flowering time is an important trait in Brassica vegetables because early flowering often leads to low yield and low quality. It is also important for oilseed rape varieties as they are divided into "winter" and "spring" types according to their different flowering times and responsiveness to vernalization. Winter types

\footnotetext{
* Correspondence: wangxw@mail.caas.net.cn

${ }^{\dagger}$ Equal contributors

'Institute of Vegetables and Flowers, Chinese Academy of Agricultural

Sciences, Beijing 100081 China

Full list of author information is available at the end of the article
}

must be exposed to cold to transition from the vegetative growth stage to the reproductive stage, while this is not necessary for the spring types, which are generally grown in shorter-season areas.

In Arabidopsis, studies of natural variation demonstrated that the vernalization requirement is largely conferred by two dominant genes, FRI and FLC [1-3]. FRI acts upstream of FLC to positively regulate FLC expression [4]. FLC encodes a MADS-box transcription factor that functions as a repressor of flowering by inhibiting downstream floral integrator genes [5-9]. Vernalization represses the expression of $F L C$ and induces flowering. The promoter and first exon of $F L C$ are sufficient to initiate the repression of $F L C$ during vernalization, while the maintenance of repression requires additional regions of the gene body [10].

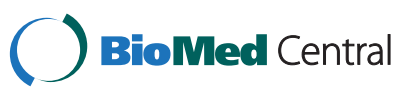


There are four copies of FLC in B. rapa [11-13]. They are located on chromosomes A10 (BraA.FLC.a, named BrFLC1), A02 (BraA.FLC.b, named BrFLC2), and A03 (BraA.FLC.c, named BrFLC3 and BraA.FLC.d, named $B r F L C 5)$ owing to polyploidy evolution [13,14]. Colinearity analysis indicated that BrFLC1, BrFLC2, and BrFLC3 are located in three $\mathrm{R}$ blocks (Xiaowu Wang et al. unpublished data), which is consistent with the three FLC copies that would be expected after a triplication event [12-15]. BrFLC5 is located between blocks I and J [16]. Multiple gene copies are thought to be responsible for dose-regulated expression, and the mechanism appears to affect variations in flowering time in Brassica crops [17]. These replicated genes may have additive effects. Depending on the specific cross studied, different alleles of the various FLC paralogs may exert different effects on flowering time. In a backcross population derived from two recombinant inbred lines, created from a cross between Per and R500, a quantitative trait locus(QTL) co-located with BrFLC1 explained more of the flowering time variation than $B r F L C 2$ [12]. It was also reported that a naturally occurring splicing variation in BrFLC1 was associated with variation in flowering time in $B$. rapa, and this locus contributed most of its effect to late flowering [18]. However, Zhao et al. [19] studied a doubled haploid (DH) population derived from a cross between pak choi and yellow sarson, and reported BrFLC2 as a candidate gene for a major QTL for flowering time and the vernalization response in $B$. rapa. The decreased transcript level of BrFLC2 upon cold treatment provided further evidence for this hypothesis.

Since there are apparent contradictions in the proposed roles of BrFLC1 and BrFLC2, we analyzed sequence variation of BrFLC1 and BrFLC2 in a large collection of $B$. rapa accessions, and the relationship between sequence variations and flowering time. Our results indicate that among the various $B$. rapa crop types, there are different genetic controls of flowering time.

\section{Results}

\section{Flowering time variation}

We determined flowering time for the germplasm collection of $159 \mathrm{~B}$. rapa accessions in two separate experiments; one in an open field in Kunming, South China, and one in a heated greenhouse in Beijing, North China. The data from the two experiments were significantly correlated with each other $\left(R^{2}=0.68, P \leq 0.01\right)$. The flowering time varied from 52 to 155 days from sowing to the opening of the first flower (days to flowering, DTF) in the open field experiment, and 42 to 150 DTF in the greenhouse experiment (Table 1, Additional file 1). Flowering time varied more within turnip rape than within the other subspecies, with a variation range of 87 days in the open field to 104 days in the greenhouse. The three yellow sarson accessions showed the narrowest variation ( 5 days in the open field, 3 days in the greenhouse). The pak choi accessions showed the greatest difference in flowering time between the two experiments, indicating that day length likely affected the timing of flowering more than vernalization, since the average day length was more than $200 \mathrm{~h}$ per month during December to April in Kunming, compared with 170-190 $\mathrm{h}$ per month in Beijing. In the open field experiment, among the vegetable-type $B$. rapa accessions, Caixin and Zicaitai flowered earlier, with an average flowering time of 82 DTF and 90 DTF, respectively, while both Chinese cabbage and pak choi flowered at 124 DTF on average. Turnips flowered latest, 142 DTF on average, followed by Wutacai (average, 133 DTF). The results from the greenhouse experiments were

Table 1 Flowering time variation among the germplasm collection of $B$. rapa

\begin{tabular}{|c|c|c|c|c|c|c|}
\hline \multirow[t]{2}{*}{ Cultivar Group } & \multirow[t]{2}{*}{ Subspecies } & \multirow[t]{2}{*}{ No. of Acc. } & \multicolumn{2}{|c|}{ Open field } & \multicolumn{2}{|c|}{ Greenhouse } \\
\hline & & & Ave. DTF & Var. range (d) & Ave. DTF & Var. range $(d)$ \\
\hline Turnip & ssp. rapa & 23 & 142 & $113-155$ & 143 & $108-150$ \\
\hline Chinese cabbage & ssp. pekinensis & 5 & 124 & $99-136$ & 118 & $69-138$ \\
\hline Pak choi & ssp. chinensis & 29 & 124 & $103-154$ & 121 & $56-150$ \\
\hline Caixin & ssp. parachinensis & 11 & 82 & $52-115$ & 70 & $50-108$ \\
\hline Broccoletto & ssp. broccoletto & 4 & 105 & 74-152 & 108 & $80-150$ \\
\hline Zicaitai & ssp. chinensis var. purpurea & 7 & 90 & $52-117$ & 97 & $55-126$ \\
\hline Mizuna & ssp. nipposinica & 1 & 142 & - & 136 & - \\
\hline Neep greens & ssp. perviridis & 2 & 123 & $115-130$ & 143 & $135-150$ \\
\hline Wutacai & ssp. narinosa & 3 & 133 & $127-139$ & 133 & $121-142$ \\
\hline Turnip rape & ssp. oleifera & 71 & 86 & $67-154$ & 85 & $46-150$ \\
\hline Yellow sarson & ssp. tricolaris & 3 & 77 & $74-79$ & 55 & $53-56$ \\
\hline
\end{tabular}

Acc., Accessions; Ave. DTF, Average days to flowering; Var., variation. 
Table 2 Nine B. rapa accessions used to sequence $B r F L C 2$ in this study

\begin{tabular}{llll}
\hline Accessions & Subspecies & Cultivar group & Flowering behavior \\
\hline HN54 & ssp. pekinensis & Chinese cabbage & annual, early \\
\hline L144 & ssp. oleifera ${ }^{\text {a }}$ & Rapid cycling & annual, very early \\
\hline L77 & ssp. pekinensis & Chinese cabbage & annual, early \\
\hline Z16 & ssp. pekinensis & Chinese cabbage & annual, early \\
\hline L143 & ssp. tricolaris & Yellow sarson & annual, early \\
\hline L203 & ssp. nipposinica & Mizuna & biannual \\
\hline CGN15202 & ssp. perviridis & Neep greens & biannual \\
\hline CGN06818 & ssp. rapa & Turnip & biannual \\
\hline L58 & ssp. parachinensis & Caixin & annual, early \\
\hline a20]. & & &
\end{tabular}

largely consistent with those obtained from the open field experiments (Table 1, Additional file 1).

\section{Nucleotide polymorphisms at the BrFLC2 gene}

Nine accessions were selected to sequence BrFLC2 (Table 2). These nine accessions represented seven $B$. rapa subspecies, and showed a wide range of variation in flowering times. For six of the nine accessions, flowering time varied from 38 to 66 DTF in the greenhouse in 2009. The other three accessions (L203, CGN06818, CGN15202) had not flowered by 90 days after sowing. To investigate the allelic variation in BrFLC2, a 1400-bp fragment was amplified from $B$. rapa genomic DNA with the primer combination of FLC2F8 in exon 4 and FLC2R6 in exon 7 (Figure 1). Sequencing of the amplified fragments from the nine selected accessions revealed that the amplified region had multiple sequence variations among the nine accessions. All of these polymorphisms were single nucleotide substitutions, with two additional insertion/deletions (InDel) in line L143, a yellow sarson accession. One was a deletion of $57 \mathrm{bp}$, started at 1851 bp of BrFLC2 (Bra028599, http://brassicadb.org/brad/) and ended at $1914 \mathrm{bp}$, across exon 4 and intron 4 . The deletion was interrupted by 5 nucleotides (TAAAT) that could not be mapped to a certain position of the reference sequence (Figure 2, Additional file 2). The other one was an insertion of $29 \mathrm{bp}$ at the position of 2430 bp located in intron 6 of BrFLC2. All the single nucleotide substitutions were synonymous. An InDel marker designated as BrFLC2InDel was developed to distinguish the 57 bp deletion across exon 4 and intron 4 and validated in the nine sequenced accessions (Figure 2).

\section{Relationship between flowering time and nucleotide polymorphisms in BrFLC2}

The BrFLC2 InDel was screened over the germplasm collection consisting of 159 accessions. The deletion allele was absent from all of the vegetable-type subspecies, but it was present as a homozygous allele in nine out of 71 B. rapa ssp. oleifera accessions and in all three of the ssp. tricolaris accessions, and as a heterozygous allele in 13 B. rapa ssp. oleifera accessions (Table 3, Additional file 1). The correlation analysis showed that the InDel polymorphism was significantly associated with variation in flowering time among the oil-type $B$. rapa accessions (Table 3). In all cases, accessions with the deletion allele flowered early, with an average flowering time measured as days to flowering (DTF) of 72 in the open field experiment and 62 DTF in the greenhouse experiment. In contrast, the accessions without this deletion (i.e. those with the functional allele) flowered significantly later $(\mathrm{P} \leq 0.001)$ with an average DTF of 90 in the open field experiment and 93 in the greenhouse experiment. The

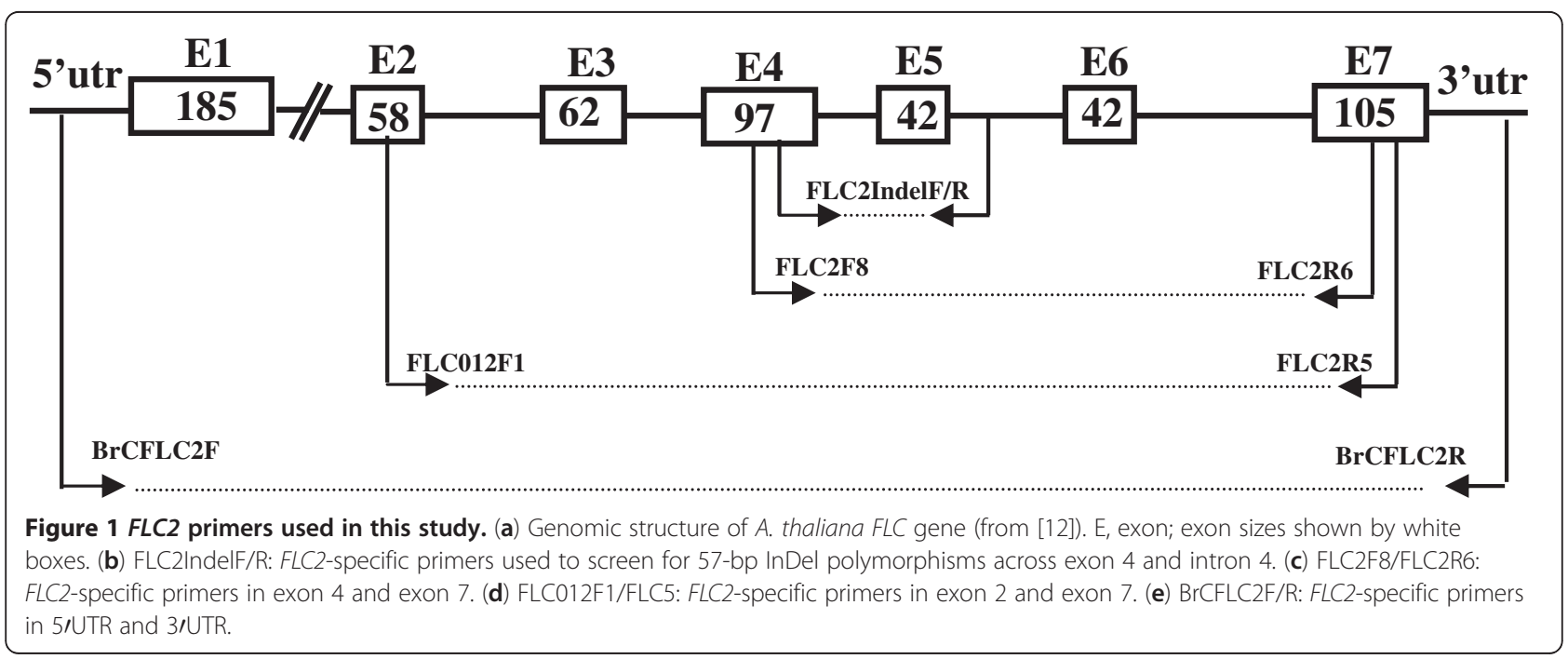




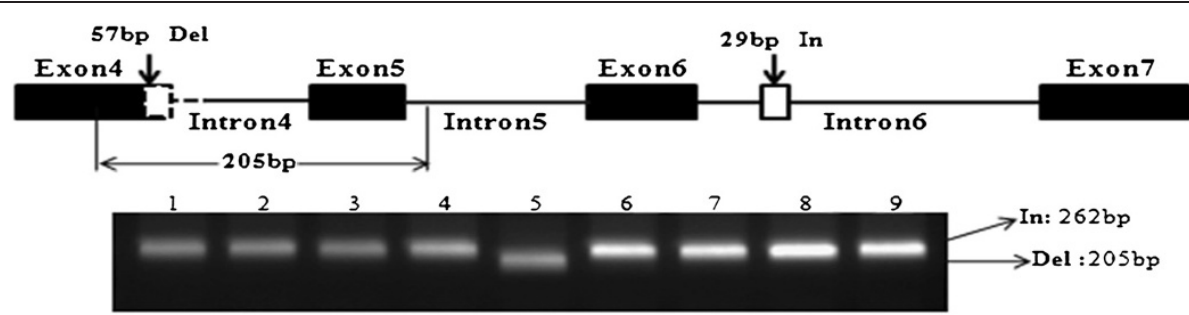

Figure 2 Schematic model of development of InDel marker used to detect 57-bp deletion mutation across exon 4 and intron 4 of BrFLC2. (a) Insertion and deletion identified in B. rapa ssp. olerferious. Del, deletion; In, insertion. Amplified region used to detect deletion mutation covered part of exon 4, intron 4, and part of exon 5. (b) Amplified fragments. In and Del represent insertion allele and deletion allele, respectively, followed by fragment size (in base pairs). Nine accessions (1-9) are listed in same order as in Table 2.

flowering time of the accessions with the heterozygous $B r F L C 2$ locus were similar to those of accessions with the homozygously mutated locus, indicating that early flowering was dominant over late flowering.

We further analyzed the 159 accessions using the previously reported BrFLC1MvaI CAPS marker, which can distinguish A/G alleles located at the splicing site of exon 4 and intron 4 of BrFLC1. The A allele results in alternative splicing, which is correlated with early flowering in B. rapa [18]. BrFLC1 allelic variation was observed in both vegetable-types and oil-types, showing A, G, and heterozygous alleles (Table 3). The flowering time in vegetable-type $B$. rapa was significantly correlated with the A/G genotype; accessions with the A genotype had an average flowering time of 108 DTF in the open field experiment and 105 DTF in the greenhouse experiment. Accessions with G genotype flowered at 132 DTF in the open field experiment and 137 DTF in the greenhouse experiment. However, there was no significant relationship between the BrFLC1 A/G genotype and variations in flowering time among oil-type $B$. rapa accessions. The mean DTF of the 38 oil-type accessions with the A allele was 85 in the open field experiment and 84 in the greenhouse experiment. In contrast, the average flowering time of 11 accessions with the $\mathrm{G}$ allele was 92 DTF in the open field experiment and 83
DTF in the greenhouse experiment. Similar to $B r F L C 2$, early flowering controlled by $B r F L C 1$ was dominant over late flowering (Table 3).

The association between flowering time and the BrFLC2 InDel alleles was also analyzed for $\mathrm{DH}$ progenies from a $\mathrm{BC}_{2}$ population, using a Chinese cabbage accession Z16 as the recurrent parent and yellow sarson accession L143 as the donor. Both parents had "A" alleles for BrFLC1, while Z16 had a BrFLC2 allele without deletions that was possibly functional, and L143 had the deletion allele of BrFLC2. Of the 120 screened DH lines, 3 had the deletion allele. We investigated the flowering phenotype of these three lines, five randomly selected lines with the functional allele, and the two parental lines. The lines with the deletion allele showed significantly shorter flowering times than those of lines with the functional allele (Table 4). The flowering times of DH lines with the functional BrFLC2 allele ranged from 83 DTF to 92 DTF, while those of lines with the mutated deletion allele ranged from 70 DTF to 80 DTF.

\section{Alternative splicing of BrFLC2}

To identify any alternative splicing of the mutated $B r F L C 2$ allele, RT-PCR was conducted with BrFLC2-specific primers (BrCFLC2F and BrCFLC2R) using the yellow sarson accession L143, which had a homozygous deletion allele.

Table 3 Flowering time variation among germplasm collection of $B$. rapa

\begin{tabular}{|c|c|c|c|c|c|c|}
\hline \multirow[t]{2}{*}{ Genotype } & \multicolumn{3}{|c|}{ Vegetables } & \multicolumn{3}{|c|}{ Oil type* } \\
\hline & No. of lines & DTF Open field & DTF Greenhouse & No. of lines & DTF Open Field & DTF Greenhouse \\
\hline \multicolumn{7}{|l|}{ BrFLC1 } \\
\hline $\bar{A}$ & 45 & $108 \pm 25 b$ & $105 \pm 45 b$ & 38 & $85 \pm 12 a$ & $84 \pm 25 a$ \\
\hline $\bar{G}$ & 37 & $132 \pm 20 a$ & $137 \pm 22 \mathrm{a}$ & 11 & $92 \pm 25 a$ & $83 \pm 33 a$ \\
\hline$\overline{\mathrm{H}}$ & 3 & $112 \pm 12 b$ & $115 \pm 35 b$ & 25 & $83 \pm 12 a$ & $83 \pm 18 \mathrm{a}$ \\
\hline \multicolumn{7}{|l|}{ BrFLC2 } \\
\hline insertion & 85 & $119 \pm 26$ & $118 \pm 30$ & 49 & $90 \pm 15 a$ & $93 \pm 23 a$ \\
\hline deletion & & & & 12 & $72 \pm 4 b$ & $62 \pm 18 b$ \\
\hline $\mathrm{H}$ & & & & 13 & $78 \pm 8 b$ & $70 \pm 12 b$ \\
\hline
\end{tabular}

DTF (days to flowering) data are presented as means \pm SE. Significant differences $(P \leq 0.001)$ between lines are indicated by different letters. *Includes ssp. oleifera and ssp. tricolaris. 
Table 4 Flowering time in $\mathrm{BC}_{2} \mathrm{DH}$ progenies with different BrFLC2 alleles

\begin{tabular}{lll}
\hline Line & BrFLC2 allele & DTF \\
\hline Z16 & In & $83 \pm 1.64 \mathrm{cde}$ \\
\hline L143 & Del & $78 \pm 3.13 \mathrm{ef}$ \\
\hline WJ10Q011 & Del & $80 \pm 2.41 \mathrm{def}$ \\
\hline WJ10Q012 & Del & $70 \pm 2.00 \mathrm{~g}$ \\
\hline WJ10Q013 & Del & $76 \pm 1.52 \mathrm{fg}$ \\
\hline WJ10Q014 & In & $85 \pm 1.00 \mathrm{bcd}$ \\
\hline WJ10Q015 & In & $89 \pm 0.00 \mathrm{abc}$ \\
\hline WJ10Q016 & In & $91 \pm 2.17 \mathrm{ab}$ \\
\hline WJ10Q017 & $\ln$ & $92 \pm 2.19 \mathrm{a}$ \\
\hline WJ10Q018 & In & $83 \pm 1.37 \mathrm{cde}$ \\
\hline
\end{tabular}

DTF (days to flowering) data are presented as means \pm SE. Significant differences $(P \leq 0.001)$ between lines are indicated by different letters.

Three fragments of different lengths were amplified from the cDNA of L143 (Figure 3). Sequencing of these fragments revealed three alternative splicing patterns. The 5 nucleotides (TAAAT) which interrupted the $57 \mathrm{bp}$ deletion were found in transcripts from all three splicing patterns. The three patterns were as follows: SPD1) 600-bp transcript -18 bp skipped at the end of exon 4 following the (TAAAT) insertion and $22 \mathrm{bp}$ retained at the end of intron 4; SPD2) 551-bp transcript-49 bp skipped at the beginning and 18 bp skipped at the end of exon 4, the retained 30 bp of exon 4 followed by the (TAAAT) insertion and $22 \mathrm{bp}$ retained at the end of intron 4; and SPD3) 686-bp transcript-same as SPD1 besides intron 3 retained. Of the 36 sequenced cDNA clones, 28 showed SPD1, six showed SPD2, and two showed SPD3. This indicated that the SPD1 was the major splicing pattern for the deletion allele of BrFLC2, while SPD2 and SPD3 were derived from SPD1.

\section{Discussion}

An InDel polymorphism across exon 4 and intron 4 of BrFLC2 was discovered in a subset of oil-type $B$. rapa accessions, including ssp. oleifera and ssp. tricolaris. In this study, we investigated its relationship with flowering time both in a collection of $B$. rapa natural accessions and in a $\mathrm{BC}_{2} \mathrm{DH}$ population.

Plants need to sense their environment and initiate flowering at the appropriate time to ensure successful fertilization and production of abundant seeds. There is considerable variation in the flowering time among, but also within, natural populations, as we observed in the present study of a $B$. rapa germplasm collection. In $A$. thaliana, the FRI gene was shown to be a major determinant of flowering time variation in the natural population through its effects on the expression of $F L C$ [21,22]. An InDel variation in the COL1 gene was reported to be correlated with variation in flowering time in $B$. nigra [23]. In $A$. thaliana, FLC encodes a MADS-box transcription

(a)

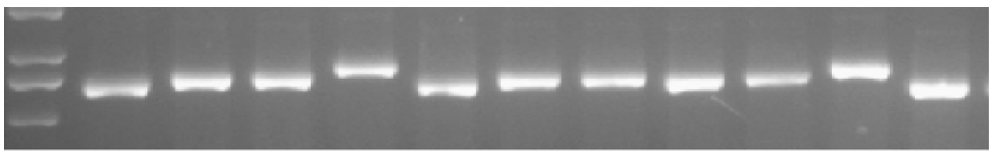

(b)

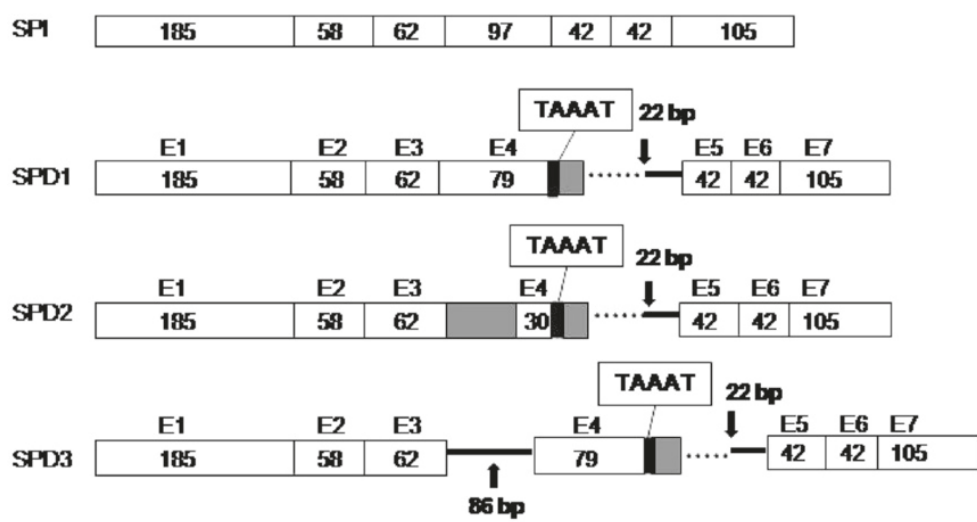

Figure 3 Sequence variation affects RNA splicing. (a) PCR fragments amplified from 11 E. coli clones transformed with BrFLC2 RT-PCR products from yellow sarson accession L143. (b) Three alternative splicing patterns identified in accession L143. SPI: constitutive splicing pattern [11]; SPD1-3: alternative splicing patterns. White boxes indicate exons; shaded box, missing parts of exon 4; dotted lines, intron parts missing from transcripts; solid lines, retained parts of introns; black bars, the 5 bp insertion (TAAAT). 
factor that acts as a dose-dependent flowering repressor $[9,22]$. Four copies of the FLC gene in B. rapa increase the potential variation in flowering time [17]. In our previous study, we identified a splicing site polymorphism Pi6 +1 (G/A) in BrFLC1 that was significantly associated with the naturally occurring variation in flowering time in $B$. rapa [18]. In that study, we examined 96 lines, six of which were oil-types. In contrast, half of the lines examined in the present study were oil-types. Because there were so few oil-types in our previous study, we did not identify that oil-type and vegetable-type $B$. rapa showed different relationships between alleles of BrFLC1 and flowering time variation. In the present study, we could not detect any effect of allelic variation in BrFLC1 $\mathrm{Pi} 6+1(\mathrm{G} / \mathrm{A})$ on the variation in flowering time for oil-type B. rapa, including ssp. oleifera and ssp. tricolaris. However, we detected an InDel polymorphism across exon 4 and intron 4 of BrFLC2 among the accessions of ssp. oleifera and ssp. tricolaris. This sequence polymorphism was not detected in any of the other vegetable-type $B$. rapa subspecies. Furthermore, this allelic variation was strongly associated with variations in flowering time in oil-type B. rapa. Zhao et al. [19] suggested that BrFLC2 was a major determinant of flowering time variation in $B$. rapa. However, there were no firm conclusions from several studies on BrFLC1 $[12,18]$ and BrFLC2 [19]. Since BrFLC1 and BrFLC2 have specific roles in controlling flowering time in different $B$. rapa groups, we deduced that there was an independent evolution of the control of flowering time, at least the control of the vernalization pathway, between oil-type $B$. rapa including ssp. oleifera and ssp. tricolaris and the other vegetable-type and turnip $B$. rapa subspecies. The oil-type $B$. rapa formed an evolutionary branch that was independent of other $B$. rapa species in an analysis of molecular phylogeny based on whole genome re-sequencing data generated from 108 accessions (Dr. Xiaowu Wang, unpublished data). This indicates that the evolutionary history of oil-type $B$. rapa is isolated from that of the vegetable-type subspecies. The fact that the deletion mutation of BrFLC2 was absent from vegetable-type $B$. rapa indicates that this mutation may have arisen after the division of oil-type from vegetable-type $B$. rapa, while the splicing site mutation of BrFLC1 may have arisen before this division and been maintained during their respective evolutions.

Relationships between naturally occurring alternative splicing variants and flowering time variation have been reported for the FLC gene in A. thaliana [24] and Capsella bursa-pastoris [25], and for BrFLC1 in B. rapa [18]. Alternative splicing variants were also reported for BrFLC5 in a biennial oilseed cultivar, although their relationship with flowering time was not addressed [12]. In the present study, we detected three alternative splicing patterns for BrFLC2 in the yellow sarson accession L143, which has a homozygous deletion allele of BrFLC2. All three alternative splicing variations led to the insertion of premature stop codons in the transcripts. The alternative splicing pattern iii of BrFLC2 has been reported by Zhao et al. [19] using DH lines derived from a cross between the same yellow sarson accession and a pak choi accession, and was deduced to be a regulatory mechanism for the differential expression of $B r F L C 2$ in response to vernalization. In the present study, the transcripts from splicing pattern iii were the minor fraction of transcripts from the deletion allele of BrFLC2. This could be due to the different cultivation conditions in the two studies, as the plants were not cold-treated in this study. A possible reason for the differential expression in response to cold treatment might be that alternative splicing transcripts were eliminated by the mRNA surveillance system. Eukaryotes have an mRNA surveillance system to eliminate the transcripts that are deliberately spliced to contain premature stop codons as a part of their intricate autoregulatory system [26].

$B$. rapa is a mesohexaploid that has undergone whole genome triplication after divergence from a common ancestor of $A$. thaliana. During the diploidization process afterwards, which involved considerable gene loss, some gene family showed preferential retention such as circadian clock genes [27], and also many of flowering time genes. It has been speculated that polyploidy and lost of the duplicated genes may have contributed to the evolution of variations in flowering time, a key component of morphological diversity [17]. After the hexaploid process, the three sub-genomes of the ancestor were partitioned into LF, MF1, and MF2 [13]. BrFLC1 is located in LF, BrFLC2 in MF2, and BrFLC3 in MF1 [13], while $B r F L C 5$ is located in the homologous region generated from an $\alpha$-duplication event that occurred before the diversification of Arabidopsis-Brassica [14]. The fact that a non-functional BrFLC1 mutation introduces early flowering time variation in vegetable-type $B$ rapa, while the non-functional $B r F L C 2$ introduces early flowering time variation in oil-type $B$. rapa, indicates that these two loci of FLC in B. rapa play different roles in different groups. It has been proposed that nonfunctionalization of duplicate genes could provide an important source of phenotypic variation [25]. We have shown that the deletion in BrFLC2 also promoted flowering in a genetic background of Chinese cabbage line Z16. However, it remains unknown why different alleles of BrFLC1 show no difference in flowering time in oiltype $B$. rapa accessions. We need to sequence all of the BrFLC1 alleles in these accessions to determine whether they contain additional mutations. Genetic redundancy provides flexibility for plants growing in changeable environments. It is also possible that the other two homologs of FLC might function to compensate for the loss of function of BrFLC1 or BrFLC2. We sequenced $B r F L C 3$ or $B r F L C 5$ using primers designed from 
sequences in exon 4 and exon 7 , respectively, and we did not identify any functional sequence variation for the nine accessions (unpublished data). However, we can not exclude the possibility that there are sequence variations located in other regions that might affect their functions. Further research on BrFLC3 in subgenome MF1 and BrFLC5 as a relic of the $\alpha$ duplication event and their influence on flowering is underway. We anticipate that they may have differentiated from, and are functionally different from, $B r F L C 1$ and BrFLC2.

\section{Conclusions}

Our results suggest that the naturally occurring deletion mutation across exon 4 and intron 4 in BrFLC2 contributes greatly to the variation in flowering time in oiltype $B$. rapa. The different relationships between $B r F L C 1$ or $B r F L C 2$ and the variation in flowering time of vegetable-type and oil-type $B$. rapa indicate that control of flowering time undergone separate evolution between these two groups.

\section{Methods}

Plant materials and flowering time evaluation

To characterize the natural variation of flowering time in B. rapa, we measured 159 accessions belonging to 11 cultivar groups (Table 1, Additional file 1). We investigated flowering time for this germplasm collection under open field conditions in Kunming, Yunnan Province, southern China, from 12 October 2009 to 1 April 2010, as well as in a greenhouse in Beijing, northern China, from 23 October 2010 to 20 March 2011. The lowest mean daily temperature in Kunming is in December and January (average, $8.2^{\circ} \mathrm{C}$ ). The day length varied between $142.7 \mathrm{~h}$ in October and $244.8 \mathrm{~h}$ in April. The day length in Beijing is 2,562-2,744 $\mathrm{h}$ per year on average. The shortest days are in winter (581.6 $\mathrm{h}$ in total during December to February), and the longest days are in spring (778.8 $\mathrm{h}$ in total from March to May). The temperature in the greenhouse varied between 15 to $25^{\circ} \mathrm{C}$ day/night, and no additional lighting was supplied.

Nine $B$. rapa accessions (Table 2) with a wide range of flowering times were used to screen for $B r F L C 2$ sequence variations. The accessions were grown in a greenhouse in Beijing in the fall of both 2008 and 2009 to investigate variations in flowering times.

A $\mathrm{BC}_{2} \mathrm{DH}$ population with 120 lines derived from a cross between the yellow sarson line L143 (R500 from Wisconsin University) and the Chinese cabbage line Z16, using Z16 as the recurrent parent, were screened for polymorphisms of the BrFLC2Indel marker. We selected five lines with the insertion allele and three lines with the deletion allele and grew them in the greenhouse in Beijing from 20 August 2010 to investigate flowering time. Five replicates were grown for each line. Flowering time was scored as the number of days from sowing to the opening of the first flower (DTF). For the nine $B$. rapa accessions that were used to sequence $B r F L C 2$, DTF was recorded as described above until $90 \mathrm{~d}$ and then those plants with no open flowers were transferred to a cold treatment. For the germplasm collection of 159 accessions grown in the open field, DTF was recorded until 155 DTF, while in the greenhouse flowering time was recorded until 150 DTF. Plants that did not have open flowers at the end of the experiment were assigned a value of 90 DTF, 155 DTF, and 150 DTF in the three respective experiments.

\section{BrFLC2 amplification}

Genomic DNA was isolated from leaf samples using the CTAB method [28]. Specific primers were designed for BrFLC2 (AY205317S1, AY205317S2). BrFLC2 was amplified by nested PCR. The outside forward primer was FLC012F1 (5/- CCTTGATCGATATGGGAAACAAC -3/) located in exon 2 and the outside reverse primer was FLCR5 (5/- TAATTAAGYAGYGGGAGAGTY AC-3/) located in exon 7. The inside forward primer was FLC2F8 (5/-GGAATCAAATTCTGATGTAAGCGTC -3/) located in exon 4 and the inside reverse primer was FLC2R6 (5/-TTTGTCCAGGTGACATCTCCATT-3/) located in exon 7 (Figure 1). The amplified fragment covered the region of exons 4-7 and the intervening introns between these exons. PCR was carried out in a total volume of $20 \mu \mathrm{l}$ containing $50 \mathrm{ng}$ template DNA, $0.5 \mu \mathrm{M}$ each primer, $200 \mu \mathrm{M}$ dNTPs, $1 \times$ PCR reaction buffer, and $1 \mathrm{U} \mathrm{Taq}$ polymerase. PCR was performed under the following conditions: the template was denatured at $94^{\circ} \mathrm{C}$ for $5 \mathrm{~min}$, followed by 35 cycles of amplification $\left(94^{\circ} \mathrm{C}\right.$ for $1 \mathrm{~min}, 56^{\circ} \mathrm{C}$ for $1 \mathrm{~min}, 72^{\circ} \mathrm{C}$ for $\left.1 \min 30 \mathrm{~s}\right)$, and a final extension at $72^{\circ} \mathrm{C}$ for $10 \mathrm{~min}$. PCR products from the nine accessions listed in Table 2 were purified by ethanol and $\mathrm{NaAc}(3 \mathrm{M}, \mathrm{pH}=5.4)$ and then cloned into the PMD-18 T vectors (Promega, http://www.promega. com) for sequencing.

\section{InDel marker analysis and CAPS marker analysis}

The forward primer FLC2IndelF (5/-GTCGACTCCCTCG TTCAGC -3/) in exon 4 and the reverse primer FLC2IndelR (5/-AGGGAAACTAATACAATACGCAA -3/) in intron 5 were designed to develop an InDel marker for $B r F L C 2$. PCR was performed under the following conditions: denaturation at $94^{\circ} \mathrm{C}$ for $3 \mathrm{~min}$, followed by 35 cycles of amplification $\left(94^{\circ} \mathrm{C}\right.$ for $45 \mathrm{~s}, 55^{\circ} \mathrm{C}$ for $45 \mathrm{~s}, 72^{\circ} \mathrm{C}$ for $1 \mathrm{~min}$ ), and a final extension at $72^{\circ} \mathrm{C}$ for $10 \mathrm{~min}$. The PCR products were fractionated on an $8.0 \%$ polyacrylamide gel to determine the genotype of the InDel marker. We used the CAPS marker for BrFLC1, FLC1-MvaI, to screen the 159 accessions as described by Yuan et al. [18]. 


\section{RNA extraction and reverse-transcriptase PCR (RT-PCR)}

Germinated seeds from the yellow sarson accession L143 were planted in pots in a growth chamber at $25 / 20^{\circ} \mathrm{C}$ (day/ night) with a 16-h light/8-h dark photoperiod. Young leaves were collected from plants 14 days after sowing. Total RNA was extracted using TRIplant Reagent (BioTeke, http:// www.bioteke.com/). First-strand cDNA was synthesized from $1 \mu \mathrm{g}$ total RNA using a cDNA synthesis kit (MBI Fermentas, http://www.fermentas.com) according to the manufacturer's instructions. Because there are four homologs of FLC in B. rapa, we designed specific primers to amplify to amplify the cDNA of BrFLC2, based on the B. rapa genome sequence (http://brassicadb.org/brad/). The specific primers were as follows: forward, BrCFLC2F (5/-CCGAACCTCAGGATCAAATT -3/) located in the 5/UTR, and reverse, BrCFLC2R (5/ -TTCACCCTTA TAGGGGAATAGTT -3/) located in the 3/UTR. We carried out RT-PCR under the following conditions: denaturation at $94^{\circ} \mathrm{C}$ for $4 \mathrm{~min}$, followed by 35 cycles of amplification $\left(94^{\circ} \mathrm{C}\right.$ for $45 \mathrm{~s}, 60^{\circ} \mathrm{C}$ for $45 \mathrm{~s}, 72^{\circ} \mathrm{C}$ for $1 \mathrm{~min}$ ), and a final extension at $72^{\circ} \mathrm{C}$ for $10 \mathrm{~min}$. The amplified products were separated on SYBR green-stained 2.0\% agarose gels. RT-PCR products were precipitated using ethanol and then dissolved in $\mathrm{ddH}_{2} \mathrm{O}$. The purified $\mathrm{RT}$-PCR product was cloned into pEASY-T1 vectors (TransGen Biotech, http://www.transgen.com) for sequencing. Escherichia coli strain DH5a (Tiangen Biotech, http://www.tiangen.com/) was transformed with these constructs and the positive colonies were selected. Plasmid DNA was isolated and sequenced using corresponding primers.

\section{Sequence analysis}

The BrFLC2 gene was sequenced using an ABI3730XL DNA analyzer (Perkin-Elmer, USA). All sequences were aligned against the published BrFLC2 sequence. Sequence alignment and analysis were conducted using MegAlign of DNASTAR.

\section{Statistical analysis}

Analysis of variance (ANOVA) and analysis of association were tested by one-way ANOVA and one-tailed Pearson correlation in SPSS version 12.0.1 (SPSS Inc., Chicago, IL, USA). ANOVA was performed with marker genotype as a factor.

\section{Additional files}

Additional file 1: Flowering time and genotype of $B r F L C 1$ and BrFLC2 in 159 accessions in B. rapa germplasm collection.

Additional file 2: Sequence of fragments with insertion/deletion across exon 4 and intron 4 amplified from nine B. rapa accessions.

\section{Abbreviations}

FLC: flowering locus C; InDel: insertion/deletion; DH: doubled haploid; DTF: days to flowering.

\section{Authors' contributions}

JW and XW designed experiments. KW, JW, and JZ conducted marker analysis and analyzed data. FC performed sequence analysis. SL and QW conducted field experiments and phenotype investigations. JW, XW, and GB drafted and revised the manuscript. All authors read and approved the final manuscript.

\section{Acknowledgments}

This work was supported by grants from the National Natural Science Foundation of China (30800753). The research was conducted in the Key Laboratory of Biology and Genetic Improvement of Horticultural Crops, Ministry of Agriculture, P.R. China and the Sino-Dutch Joint Lab of Horticultural Genomics Technology, Beijing. The authors gratefully acknowledge funding from the European Community financial participation under the Seventh Framework Programme for Research, Technological Development and Demonstration Activities, for the Integrated Project NUE-CROPS FP7-CP-IP 222645. The views expressed in this publication are the sole responsibility of the authors and do not necessarily reflect the views of the European Commission. Neither the European Commission nor any person acting on behalf of the Commission is responsible for the use that might be made of the information contained herein.

\section{Author details}

${ }^{1}$ Institute of Vegetables and Flowers, Chinese Academy of Agricultural Sciences, Beijing 100081 China. ${ }^{2}$ Institute of Horticultural Science, Yunnan Academy of Agricultural Sciences, Kunming 650205 China. ${ }^{3}$ Laboratory of Plant Breeding, Wageningen University, Droevendaalsesteeg 1, 6708 PB Wageningen, The Netherlands.

Received: 10 February 2012 Accepted: 14 August 2012 Published: 28 August 2012

\section{References}

1. Cao J, Schneeberger K, Ossowski S, Gunther T, Bender S, Fitz J, Koenig D, Lanz C, Stegle O, Lippert C, Wang X, Ott F, Muller J, Alonso-Blanco C, Borgwardt K, Schmid KJ, Weigel D: Whole-genome sequencing of multiple Arabidopsis thaliana populations. Nat Genet 2011, 43:956-963.

2. Koornneef M, Alonso-Blanco C, Blankestijn-de Vries H, Hanhart CJ, Peeters AJM: Genetic Interactions Among Late-Flowering Mutants of Arabidopsis. Genetics 1998, 148:885-892.

3. Koornneef M, Blankestijn-de Vries H, Hanhart C, Soppe W, Peeters T: The phenotype of some late-flowering mutants is enhanced by a locus on chromosome 5 that is not effective in the Landsberg erecta wild-type. Plant J 1994, 6:911-919.

4. Michaels SD, Amasino RM: Loss of FLOWERING LOCUS C activity eliminates the late-flowering phenotype of FRIGIDA and autonomous pathway mutations but not responsiveness to vernalization. Plant Cell 2001, 13:935-942

5. Fornara F, de Montaigu A, Coupland G: SnapShot: Control of Flowering in Arabidopsis. Cell 2010, 141:550-550. e552.

6. Helliwell CA, Wood CC, Robertson M, James Peacock W, Dennis ES: The Arabidopsis FLC protein interacts directly in vivo with SOC1 and FT chromatin and is part of a high-molecular-weight protein complex. Plant J 2006, 46:183-192.

7. Michaels SD, Amasino RM: FLOWERING LOCUS C encodes a novel MADS domain protein that acts as a repressor of flowering. Plant Cell 1999, 11:949-956

8. Searle I, He Y, Turck F, Vincent C, Fornara F, Krober S, Amasino RA, Coupland $\mathrm{G}$ : The transcription factor FLC confers a flowering response to vernalization by repressing meristem competence and systemic signaling in Arabidopsis. Genes \& Dev 2006, 20:898-912.

9. Sheldon CC, Rouse DT, Finnegan EJ, Peacock WJ, Dennis ES: The molecular basis of vernalization: The central role of FLOWERING LOCUS C (FLC). PNAS 2000, 97:3753-3758.

10. Helliwell CA, Robertson M, Finnegan EJ, Buzas DM, Dennis ES: Vernalization-repression of Arabidopsis FLC requires promoter sequences but not antisense transcripts. PLOS ONE 2011, 6(6):e21513.

11. Kim S-Y, Park B-S, Kwon S-J, Kim J, Lim M-H, Park Y-D, Kim DY, Suh S-C, Jin Y-M, Ahn JH, Lee Y-H: Delayed flowering time in Arabidopsis and Brassica rapa by the overexpression of FLOWERING LOCUS C (FLC) homologs 
isolated from Chinese cabbage (Brassica rapa L. ssp. pekinensis). Plant Cell Rep 2007, 26:327-336.

12. Schranz ME, Quijada P, Sung S-B, Lukens L, Amasino R, Osborn TC: Characterization and effects of the replicated flowering time gene $F L C$ in Brassica rapa. Genetics 2002, 162:1457-1468.

13. Wang X, Wang H, Wang J, Sun R, Wu J, Liu S, Bai Y, Mun J-H, Bancroft I, Cheng F, Huang S, Li X, Hua W, Wang J, Wang X, Freeling M, Pires JC, Paterson AH, Chalhoub B, Wang B, Hayward A, Sharpe AG, Park B-S, Weisshaar B, Liu B, Li B, Liu B, Tong C, Song C, Duran C, Peng C, Geng C, Koh C, Lin C, Edwards D, Mu D, Shen D, Soumpourou E, Li F, Fraser F, Conant G, Lassalle G, King GJ, Bonnema G, Tang H, Wang H, Belcram H, Zhou H, Hirakawa H, Abe H, Guo H, Wang H, Jin H, Parkin IAP, Batley J, Kim J-S, Just J, Li J, Xu J, Deng J, Kim JA, Li J, Yu J, Meng J, Wang J, Min J, Poulain J, Hatakeyama K, Wu K, Wang L, Fang L, Trick M, Links MG, Zhao M, Jin M, Ramchiary N, Drou N, Berkman PJ, Cai Q, Huang Q, Li R, Tabata S, Cheng S, Zhang S, Zhang S, Huang S, Sato S, Sun S, Kwon S-J, Choi S-R, Lee T-H, Fan W, Zhao X, Tan X, Xu X, Wang Y, Qiu Y, Yin Y, Li Y, Du Y, Liao Y, Lim Y, Narusaka Y, Wang Y, Wang Z, Li Z, Wang Z, Xiong Z, Zhang Z: The genome of the mesopolyploid crop species Brassica rapa. Nat Genet 2011, 43:1035-1039.

14. Yang T-J, Kim JS, Kwon S-J, Lim K-B, Choi B-S, Kim J-A, Jin M, Park JY, Lim M-H, Kim H-I, Lim YP, Kang JJ, Hong J-H, Kim C-B, Bhak J, Bancroft I, Park $B-S$ : Sequence-level analysis of the diploidization process in the triplicated FLOWERING LOCUS C region of Brassica rapa. Plant Cell 2006, 18:1339-1347.

15. Osborn TC, Kole C, Parkin IAP, Sharpe AG, Kuiper M, Lydiate DJ, Trick M: Comparison of flowering time genes in Brassica rapa, B. napus and Arabidopsis thaliana. Genetics 1997, 146:1123-1129.

16. Feng C, Shengyi L, Jian W, Lu F, Silong S, Bo L, Pingxia L, Wei H, Xiaowu W: $B R A D$, the genetics and genomics database for Brassica plants. BMC Plant Biology 2011, 11:136.

17. Osborn TC: The contribution of polyploidy to variation in Brassica species. Physiol Plantarum 2004, 121:531-536.

18. Yuan Y-X, WU J, Sun R-F, Zhang X-W, Xu D-H, Bonnema G, Wang X-W: A naturally occurring splicing site mutation in the Brassica rapa $F L C 1$ gene is associated with variation in flowering time. J Exp Bot 2009, 60:1299-1308.

19. Zhao J, Kulkarni V, Liu N, Pino Del Carpio D, Bucher J, Bonnema G: BrFLC2 (FLOWERING LOCUS $C$ ) as a candidate gene for a vernalization response QTL in Brassica rapa. J Exp Bot 2010, 61:1817-1825.

20. Zhao J, Wang X, Deng B, Lou P, Wu J, Sun R, Xu Z, Vromans J, Koornneef $M$, Bonnema G: Genetic relationships within Brassica rapa as inferred from AFLP fingerprints. Theor App/ Genet 2005, 110:1301-1314.

21. Johanson U, West J, Lister C, Michaels S, Amasino R, Dean C: Molecular analysis of $F R I G I D A$, a major determinant of natural variation in Arabidopsis flowering time. Science 2000, 290:344-347.

22. Shindo C, Aranzana MJ, Lister C, Baxter C, Nicholls C, Nordborg M, Dean C Role of FRIGIDA and FLOWERING LOCUS C in Determining Variation in Flowering Time of Arabidopsis. Plant Physiology 2005, 138:1163-1173.

23. Osterberg MK, Shavorskaya O, Lascoux M, Lagercrantz U: Naturally occurring indel variation in the Brassica nigra COL1 gene is associated with variation in flowering time. Genetics 2002, 161:299-306.

24. Caicedo AL, Stinchcombe JR, Olsen KM, Schmitt J, Purugganan MD: Epistatic interaction between Arabidopsis $F R I$ and $F L C$ flowering time genes generates a latitudinal cline in a life history trait. PNAS 2004, 101:15670-15675.

25. Slotte T, Huang H-R, Holm K, Ceplitis A, Onge KS, Chen J, Lagercrantz U, Lascoux M: Splicing variation at a FLOWERING LOCUS C homolog is Associated with flowering time variation in the tetraploid Capsella bursa-pastoris. Genetics 2009, 183:337-345.

26. Brenton RG: Alternative splicing: increasing diversity in the proteomic world. Trends Genet 2001, 17:100-107.

27. Lou P, Wu J, Cheng F, Cressman LG, Wang X, McClung CR: Preferential Retention of circadian clock genes during diploidization following whole genome triplication in Brassica rapa. Plant Cell 2012, 24:2415-2426.

28. Wang X, Lou P, Bonnema G, Yang B, He H, Zhang Y, Fang Z: Linkage mapping of a dominant male sterility gene Ms-cd1 in Brassica oleracea. Genome 2005, 48:848-854.

doi:10.1186/1471-2229-12-151

Cite this article as: Wu et al:: A naturally occurring InDel variation in BraA.FLC.b (BrFLC2) associated with flowering time variation in Brassica rapa. BMC Plant Biology 2012 12:151.

\section{Submit your next manuscript to BioMed Central and take full advantage of:}

- Convenient online submission

- Thorough peer review

- No space constraints or color figure charges

- Immediate publication on acceptance

- Inclusion in PubMed, CAS, Scopus and Google Scholar

- Research which is freely available for redistribution 